\title{
Notes
}

\section{Surface Diluent Effect on the Heterogeneous Electron-transfer Kinetics of PQQ Self-assembled Monolayers}

\author{
Heejeong Hong, Wonchoul Park, Sunghyun Kim,* and Hyung Joo Kim ${ }^{\dagger}$ \\ Department of Bioscience and Biotechnology, Konkuk University, Seoul 143-701, Korea. *E-mail: skim100@konkuk.ac.kr \\ ${ }^{\dagger}$ Department of Microbial Engineering, Konkuk University, Seoul 143-701, Korea \\ Received December 17, 2009, Accepted March 26, 2010
}

Key Words: Heterogeneous electron transfer, Pyrroloquinoline quinone, Diluent effect, Proton-coulpled reaction

Self-assembled monolayers (SAMs) ${ }^{1}$ have been extensively used as a model system to study long-range electron transfer (ET) kinetics between an electrode and a redox center. They form molecularly flat, well organized surfaces with minimal defects. This feature makes a SAM surface an ideal platform on which fundamental issues on the heterogeneous ET kinetics could be addressed. Other advantages are that complications arising from double layer effect or diffusion process can be avoided. Many systems have been studied. They include ruthenium or osmium complexes, ${ }^{2,3}$ ferrocene, ${ }^{4,5}$ viologen, ${ }^{6}$ azobenzene, ${ }^{7,8}$ and various quinone systems. ${ }^{9-14}$ Quinones are important as they play an important role in biological systems. ${ }^{15}$ Focus has mostly been made on the evaluation of the tunneling barrier constant $(\beta)$ and the dependency of ET rate constant on the structure of bridges connecting an electrode and an electroactive center. Although it seems that there is a general agreement on the $\beta$ value of $c a$. $1.0 \AA^{-1}$ per $\mathrm{CH}_{2}$ unit, different $\beta$ values have been reported for different quinines. ${ }^{9,16}$ The heterogeneous ET rate constant is greatly affected by the nature of bridging molecules. Conjugated bridges give higher ET rate constant compared to unconjugated systems. ${ }^{17}$ Also a double-bond bridge gives the fastest ET compared to single- or triple-bond bridges. ${ }^{18}$

In this work, we have studied effects of electroinactive diluents that were coadsorbed with an electroactive SAM on the ET kinetics. Pyrroloquinoline quinone (PQQ) was chosen as an electroactive group. PQQ is found in many dehydrogenases acting as a redox cofactor. ${ }^{19,20} \mathrm{PQQ}$ has a unique heterocyclic $o$-quinone structure having three carboxylic groups (Scheme 1).

Previously we have shown that the ET kinetics of PQQ was greatly affected by the presence of diluents of fixed chain leng-<smiles>O=C(O)C1=CC(C(=O)O)NC2=C1C(=O)C(=O)c1cc(C(=O)O)[nH]c12</smiles>

Scheme 1. Structure of PQQ th. ${ }^{21}$ Diluents affected ET rate constants by lessening the lateral interaction between PQQ groups. This work is an extension of the previous study to cover the effect of different alkyl chain lengths of diluents on the ET reactions of coadsorbed PQQ.

\section{Experimental Section}

Materials and reagents. A gold wire $(99.999 \%, 0.5 \mathrm{~mm}$ diameter) was obtained from Johnson Matthey (UK). Pyrroloquinoline quinine, alkanethiols (Sigma-Aldrich, USA) and an aminoalkanethiol ( $\left(\mathrm{NH}_{2}\left(\mathrm{CH}_{2}\right)_{6} \mathrm{SH}\right.$, Dojindo, Japan) were used as received. All other chemicals were of reagent grade and used without purification.

Monolayer preparation. Working electrodes were made of Au beads ( $c a .2 .1-2.4 \mathrm{~mm}$ in diameter) formed at the tip of a gold wire ( $0.5 \mathrm{~mm}$ diameter) by melting it in oxygen-gas flame. These bead electrodes were cleaned in a hot "piranha" solution (3:1 mixture of concentrated $\mathrm{H}_{2} \mathrm{SO}_{4}$ and $30 \% \mathrm{H}_{2} \mathrm{O}_{2}$ ) for $10 \mathrm{~min}$. After rinsing with copious amount of deionized water, they were electrochemically cleaned by potential cycling in $0.5 \mathrm{M}$ $\mathrm{H}_{2} \mathrm{SO}_{4}$ in the potential range of -0.20 and $1.50 \mathrm{~V} v$ s. SCE until a typical voltammogram of clean gold was obtained. This way the surface was maintained very smooth and quite reproducible data could be obtained.

The roughness factor was measured using $\mathrm{Ru}\left(\mathrm{NH}_{3}\right)_{6}{ }^{3+}$. The actual surface area obtained from the slope of the linear plot of cathodic peak current versus (scan rate) ${ }^{1 / 2}$ for the reversible reduction was compared with a geometrical area. For the calculation, diffusion coefficient of $7.5 \times 10^{-6} \mathrm{~cm}^{2} \mathrm{~s}^{-1}$ at $25^{\circ} \mathrm{C}$ was used. $^{22}$ The roughness factor was between 1.1 and 1.2.

Fig. 1 shows a series of surface modification steps for a mixed monolayer preparation. A gold bead was thoroughly rinsed with deionized water and ethanol, and dried under nitrogen. Then the electrode was immersed in 1:1 ethanolic mixture of $5 \mathrm{mM}$ of $\mathrm{NH}_{2}\left(\mathrm{CH}_{2}\right)_{6} \mathrm{SH}$ and $5 \mathrm{mM}$ of $\mathrm{CH}_{3}\left(\mathrm{CH}_{2}\right)_{\mathrm{n}-1} \mathrm{SH}(\mathrm{n}=4$, $6,8,11)$ for $2 \mathrm{~h}$. Physically adsorbed compound was removed by rinsing with fresh deionized water. The electrode was treated in solution containing $3 \mathrm{mM}$ of PQQ and $10 \mathrm{mM}$ of 1-ethyl-3-(3dimethylaminopropyl)carbodiimide (EDC) in $10 \mathrm{mM}$ HEPES 


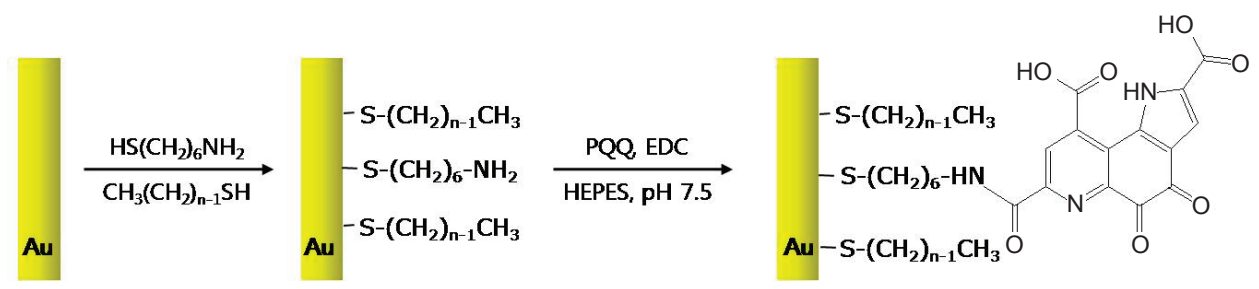

Figure 1. Surface modification steps for the formation of mixed monolayers of PQQ and alkanethiols. n= 4, 6, 8, 11 .

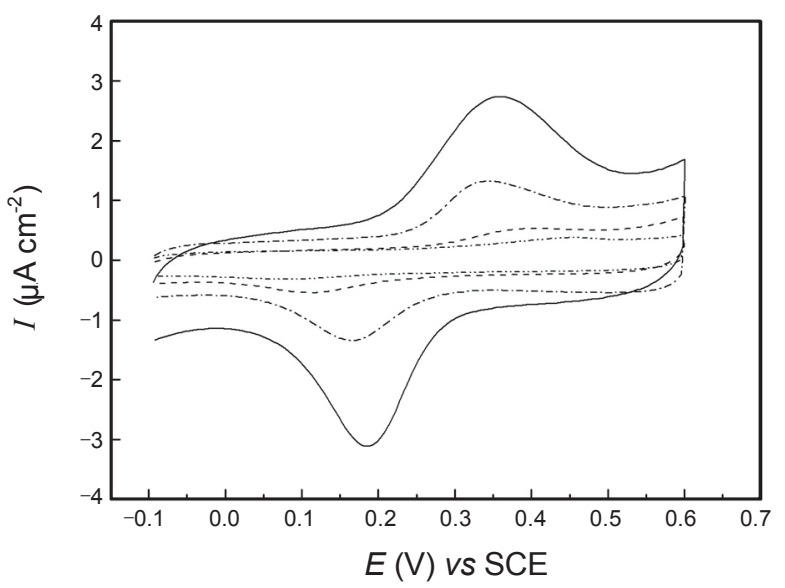

Figure 2. Cyclic voltammograms of $\mathrm{PQQ}-\mathrm{NH}\left(\mathrm{CH}_{2}\right)_{6} \mathrm{SH}$ monolayers on gold diluted by butanethiol (solid), hexanethiol (solid dot), octanethiol (dashed), and undecanethiol (solid dot dot) in $0.1 \mathrm{M} \mathrm{HClO}_{4}$. Scan rate $=0.1 \mathrm{~V} \mathrm{~s}^{-1}$.

Table 1. Peak potentials and surface coverage of PQQ-NH( $\left(\mathrm{CH}_{2}\right)_{6} \mathrm{SH}$ diluted by $\mathrm{CH}_{3}\left(\mathrm{CH}_{2}\right)_{\mathrm{n}-1} \mathrm{SH}(\mathrm{n}=4,6,8,11)$ on gold

\begin{tabular}{ccccccc}
\hline $\mathrm{n}$ & $E_{\mathrm{pa}}(\mathrm{V})$ & $E_{\mathrm{pc}}(\mathrm{V})$ & $\Delta E_{\mathrm{p}}(\mathrm{V})$ & $\Delta E_{\text {fwhm }}(\mathrm{V})$ & $\left.\Gamma(\mathrm{nmol} \mathrm{cm})^{-2}\right)$ & $\Gamma / \Gamma_{\text {full }}$ \\
\hline 4 & 0.360 & 0.185 & 0.175 & 0.120 & 0.14 & 0.61 \\
6 & 0.342 & 0.167 & 0.175 & 0.122 & 0.067 & 0.29 \\
8 & 0.397 & 0.111 & 0.286 & 0.126 & 0.037 & 0.16 \\
11 & 0.455 & 0.086 & 0.369 & 0.159 & 0.019 & 0.082 \\
\hline
\end{tabular}

buffer $(\mathrm{pH}=7.5)$ for $1 \mathrm{~h}$ to attach PQQ on the top of the amine group. Thus prepared PQQ-modified electrode was again rinsed in deionized water to remove the physically adsorbed PQQ before voltammetric study.

Measurements. Electrochemical measurements were carried out in a single compartment cell using a three-electrode system composed of a Au bead, a platinum wire, and SCE as working, counter, and reference electrodes, respectively. Voltammetric experiments were performed with a potentiostat (Autolab PGSTAT 30, ECO CHEM). All the solutions were prepared with deionized water with resistivity of $18 \mathrm{M} \Omega \mathrm{cm}$ or more purified by AquaMax-Ultra system (Younglin, Korea) and deaerated by purging with nitrogen. All measurements were carried out at room temperature.

\section{Results and Discussion}

Electrochemistry of mixed monolayers. Fig. 2 shows typical cyclic voltammograms (CVs) of PQQ-NH( $\left.\mathrm{CH}_{2}\right)_{6} \mathrm{SH}$ diluted

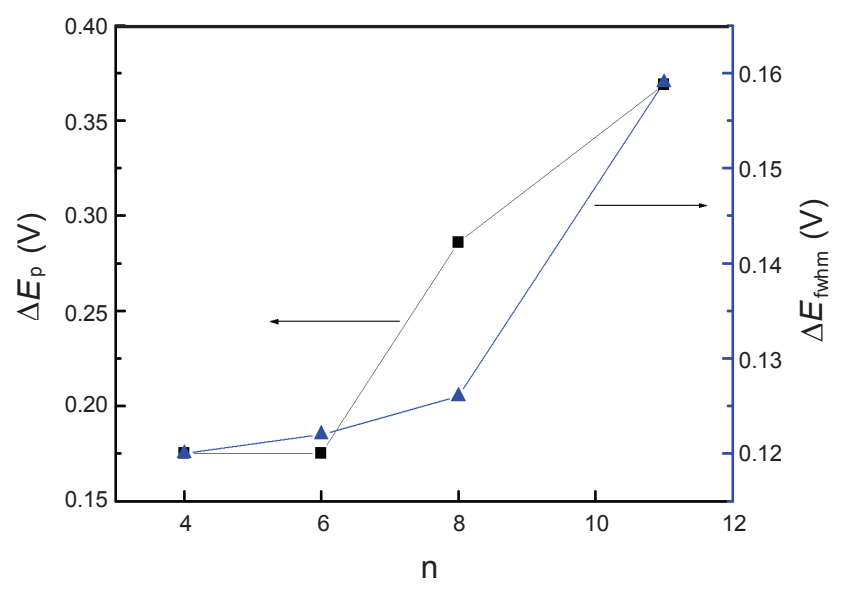

Figure 3. Plot of $\Delta E_{\mathrm{p}}(\boldsymbol{\square})$ and $\Delta E_{\mathrm{fwhm}}(\boldsymbol{\Delta}) v s$ number of carbons (n) in diluent molecules. Data were obtained from Fig. 2.

by different alkanethiols, $\mathrm{CH}_{3}\left(\mathrm{CH}_{2}\right)_{\mathrm{n}-1} \mathrm{SH}(\mathrm{n}=4,6,8,11)$ on gold. The redox waves found at $c a .0 .35$ and $0.18 \mathrm{~V}$ are due to the two-proton, two-electron process, $\mathrm{PQQ}+2 \mathrm{H}^{+}+2 \mathrm{e}^{-} \rightarrow$ $\mathrm{PQQH}_{2}{ }^{23}$ The peak currents in all four cases were linearly proportional to the scan rate indicating that PQQ was securely confined on the surface, and unchanged over the potential cycling. The surface coverage $(\Gamma)$ of PQQ was calculated from the charges under the voltammetric waves. Although mixed monolayers were prepared from 1:1 ethanolic mixture, the $\Gamma$ values are less than half of that of a full monolayer $\left(\Gamma_{f u l l}\right)$, except for $\mathrm{n}=4$ (Table 1). The coverage decrease with the increase in alkyl chain length is ascribed to the fact that alkanethiols of a longer chain length tend to form a more well-ordered and wellpacked monolayer than those of a shorter chain length. For $\mathrm{n}=$ 11 , only $8.5 \%$ of the surface is covered with PQQ. The coverage of a full monolayer was obtained without dilution.

Some voltammetric characteristics should be noted. Reversibility depends on the chain length of a diluent. $\Delta E_{\mathrm{p}}$ increases with increase in the chain length (Fig. 3). $\Delta E_{\mathrm{p}}$ for $\mathrm{n}=4$ and 6 was $175 \mathrm{mV}$ but it increased to 286 and $369 \mathrm{mV}$ for $\mathrm{n}=8$ and 11 , respectively. Full width at half maximum $\left(\Delta E_{\text {fwhm }}\right)$ can also be an indicator for the reversibility. As the chain length increases from 4 to $11, \Delta E_{\text {fwhm }}$ increases from 120 to $159 \mathrm{mV}$ accordingly. The increasing irreversibility with the chain length of diluents is surprising because it is expected that electron transfer kinetics is essentially independent of the presence of diluents under ideal conditions. With lateral interactions between electroactive head groups present, reversibility would be affected. ${ }^{24}$ Less 
Table 2. Electrochemical kinetic parameters of $\mathrm{PQQ}-\mathrm{NH}\left(\mathrm{CH}_{2}\right)_{6} \mathrm{~S} / \mathrm{Au}$ diluted by $\mathrm{CH}_{3}\left(\mathrm{CH}_{2}\right)_{\mathrm{n}-1} \mathrm{SH}(\mathrm{n}=4,6,8,11)$ in $0.1 \mathrm{M} \mathrm{HClO}_{4}$

\begin{tabular}{cccc}
\hline $\mathrm{n}$ & $\alpha \mathrm{n}$ & $(1-\alpha) \mathrm{n}$ & $\log k_{\text {app }}\left(\mathrm{s}^{-1}\right)$ \\
\hline 4 & 1.22 & 0.78 & -1.98 \\
6 & 1.28 & 0.85 & -2.26 \\
8 & 0.95 & 0.53 & -2.57 \\
11 & 0.83 & 0.69 & -4.10 \\
\hline
\end{tabular}

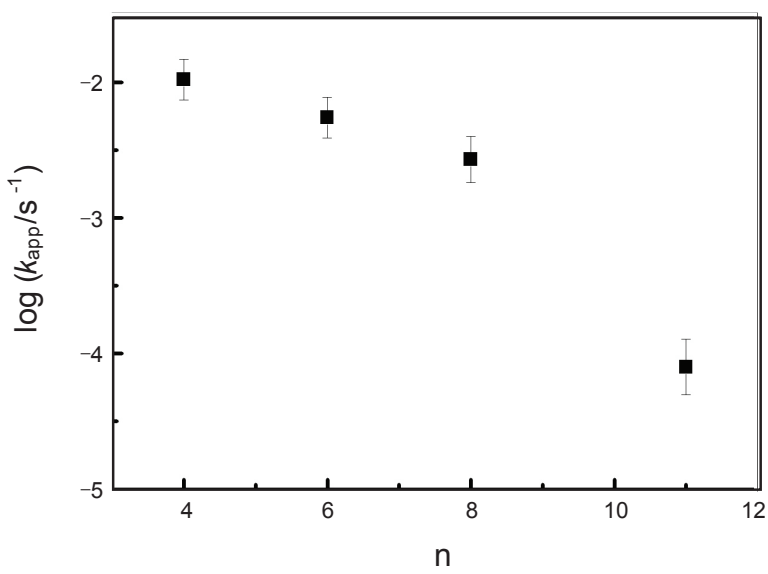

Figure 4. Dependence of apparent rate constant on the number of carbons (n) in diluent molecules.

repulsive lateral interaction upon dilution results in improved reversibility. The present results, however, shows exactly the opposite. More dilution by using a longer alkanethiol caused larger $\Delta E_{\mathrm{p}}$ and $\Delta E_{\mathrm{fwhm}}$. If the dilution only affects lateral interactions, more dilution would have resulted in better reversibility. This strongly implies that there must be other factors influencing electrochemical reactions of PQQ.

In their mixed monolayer experiments for naphthoquinone SAMs, Bulovas et al. ${ }^{25}$ have shown that proton accessibility to the quinone group played an important role in the electrochemical response. When a diluent was long enough to cover the quinone group preventing proton access to the surface from the bulk, a broad, ill-defined voltammograms were resulted. However, when a carboxyl group-terminated thiol was used, reversibility was recovered, indicating that protons from dissociation of carboxyl groups participated in the reaction. The same argument may hold for our case. The presence of a diluent makes local pH around PQQ moiety different from that of the bulk, hindering protons from having access to the surface. This hindering effect will be greater for the diluent having a longer alkyl chain length. For $n=4$ or 6 , the PQQ moiety is well above the background diluents so that the voltammetric features are not much affected. For $\mathrm{n}=8$ and 11, however, proton accessibility is much hindered, causing a local $\mathrm{pH}$ around PQQ to be higher than that of the bulk. This hindering effect becomes more and more pronounced and eventually affects electron transfer kinetics as the chain length increases.

Electron transfer kinetics. Finite electron transfer rate causes peak potential shift with scan rate. According to Laviron, ${ }^{26}$ standard rate constant and the electron transfer coefficient could be extracted from the cyclic voltammograms by the following equations.

$$
\begin{aligned}
& E_{\mathrm{pc}}=E_{\mathrm{c}}{ }^{{ }^{1}}-(R T / \alpha n F) \ln \left[\alpha n F v_{\mathrm{c}} / R T k_{\mathrm{app}}\right] \\
& E_{\mathrm{pa}}=E_{\mathrm{a}}{ }^{{ }^{\prime}}-(R T /(1-\alpha) n F) \ln \left[(1-\alpha) n F v_{\mathrm{a}} / R T k_{\mathrm{app}}\right]
\end{aligned}
$$

where $\alpha$ is the transfer coefficient, $v$ the sweep rate, $k_{\text {app }}$ the apparent rate constant, and $R, T$, and $F$ have their usual meanings. $v_{\mathrm{c}}$ and $v_{\mathrm{a}}$ are the critical scan rates obtained by extrapolating the linear portion of the $E_{\mathrm{p}} \mathrm{vs} \ln (v)$ plots to the formal cathodic and anodic potentials $E_{\mathrm{c}}{ }^{{ }_{1}}$ and $E_{\mathrm{a}}{ }^{{ }^{\prime}}$. The slopes of the linear portion of the $E_{\mathrm{p}} v s . \ln (v)$ curves are $R T / \alpha n F$ and $R T /(1-\alpha)$ $n F$ for the cathodic and anodic branches, respectively. $\alpha$ and $(1-\alpha) n$ values obtained from each slope were substituted to

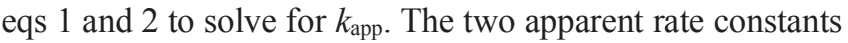
obtained in this way were averaged. Table 2 lists $\alpha$ and $(1-\alpha) n$ values along with $k_{\text {app }}$ for different chain lengths. $\alpha$ and (1- $\left.\alpha\right) n$ values are not equal, indicating the energy barrier for the redox reactions of PQQ is not symmetrical regardless of diluents.

Fig. 4 shows the plot of the logarithmic heterogeneous apparent rate constant as a function of the number of carbons $(n)$ in diluent molecules. With the increase in the chain length, the electron transfer rate becomes slower, which is the cause of the voltammetric irreversibility (see above). A slight decrease in $k_{\text {app }}$ up to $n=8$ underwent abrupt drop for $n=11$. This can be explained in terms of proton availability. The total length of PQQ-NH( $\left(\mathrm{CH}_{2}\right)_{6} \mathrm{SH}$ is $20.6 \AA$. The $o$-quinone part of PQQ is about $13 \AA$ above the surface so that butanethiol $(6.5 \AA)$ and hexanethiols (9.8 $\AA$ ) cannot effectively hinder the proton access to the PQQ moiety. A slight decrease in $k_{\text {app }}$ may indicate that PQQ-NH $\left(\mathrm{CH}_{2}\right)_{6} \mathrm{SH}$ is little bent. When an octanethiol (12.3 $\AA$ ) was used, proton availability is believed to be somewhat limited, making $k_{\text {app }}$ further decrease.

It has been well documented that proton concentration affects apparent rate constant when electron transfer is coupled with protons. Laviron ${ }^{27-30}$ formulated a nine-member square scheme for two-electron, two-proton reactions for quinone/hydroquinone redox reactions, assuming that protonation is at equilibrium. Later Finklea ${ }^{31}$ extended Laviron's work to include electron transfer coefficients varying with applied potential. The theory predicts a ' $\mathrm{W}$ '-shape curve when the rate constant is plotted against $\mathrm{pH}$ with two minima near neutral $\mathrm{pHs}$. Thus higher local proton concentration should give rise to higher rate constant in an acidic branch. The exact evaluation of local $\mathrm{pH}$, however, is possible only when all $\operatorname{six} \mathrm{p} K_{\mathrm{a}}$ values and six formal potentials for quinone/hydroquinone are known. ${ }^{32}$ Lower rate constants for longer diluents clearly indicate lower proton concentration around the PQQ moiety due to hydrophobic environment of alkyl chains. Although it is not possible to obtain exact local proton concentration, it may be at least two orders of magnitude lower for undecanethiol than for butanethiol, estimated from the apparent rate constants.

In this paper, we have formed a PQQ monolayer diluted by alkanethiols of different chain length and showed electrochemical reversibility and electron transfer rate constant are greatly affected by the local proton concentration near the electroactive 
group when protons are coupled with electrochemical reactions. By blocking the proton access to the PQQ moiety, the redox reaction of PQQ becomes irreversible and electron transfer rate slower accordingly.

Acknowledgments. This work was supported by the Korea Research Foundation Grant funded by the Korean Government (MOEHRD, Basic Research Promotion Fund) (KRF-2008313-C00413).

\section{References}

1. Ulman, A. An Introduction to Ultrathin Organic Films: From Langmuir-Blodgett to Self-Assembly; Academic Press: San Diego, 1991.

2. Weaver, M. J.; Li, T. J. Am. Chem. Soc. 1984, 106, 6107.

3. Foster, R. J.; Faulkner, L. R. J. Am. Chem. Soc. 1994, 116, 5444.

4. Chidsey, C. D. E. Science 1991, 51, 919.

5. Smalley, J. F.; Feldberg, S. W.; Chidsey, C. D. E.; Linford, M. R.; Newton, M. D.; Liu, Y.-P. J. Phys. Chem. 1995, 99, 1314.

6. Finklea,H. O.; Hanshew, D. D. J. Am. Chem. Soc. 1992, 114, 3173.

7. Yu, H. Z.; Shao, H. B.; Luo, Y.; Zhang, H. L.; Liu, Z. F. Langmuir 1997, 13, 5774.

8. Wang, Y. Q.; Yu, H. Z.; Cheng, J. Z.; Zhao, J. W.; Cai, S. M.; Liu, Z. F. Langmuir 1996, 12, 5466.

9. Mukae, F.; Takemura, H.; Takehara, K. Bull. Chem. Soc. Jpn. 1996, 69, 2461.

10. Zhang, L.; Lu, T.; Gokel, G. W.; Kaifer, A. E. Langmuir 1993, 9 , 786.

11. Hong, H.-G.; Park, W. Langmuir 2001, 17, 2485.

12. Hong, H.-G.; Park, W.; Yu, E. J. Electroanal. Chem. 1999, 476,
177.

13. Hong, H.-G.; Park, W.; Yu, E. Bull. Korean Chem. Soc. 2000, 21 , 23.

14. Tramell, S. A.; Lowry, D. A.; Seferos, D. S.; Moore, M.; Bazan, G. D.; Lebedev, N. J. Electroanal. Chem. 2007, 606, 33.

15. Chambers, J. Q. The Chemistry of the Quinonoid Compounds; Patai, S., Ed.; Wiley: New York, 1974; 737-792.

16. Nagata, M.; Konda, M.; Suemori, Y.; Ochiai, T.; Dewa, T.; Ohtsuka, T.; Nango, M. Colloids Surf. B: Biointerfaces 2008, 64, 16.

17. Trammell, S. A.; Seferos, D. S.; Moore, M.; Lowy, D. A.; Bazan, G. C.; Kushmerick, J. G.; Lebedev, N. Langmuir 2007, 23, 942.

18. Trammell, S. A.; Moore, M.; Schull, T. L.; Lebedev, N. J. Electroanal. Chem. 2009, 628, 125.

19. Duine, J. A. Eur. J. Biochem. 1991, 200, 271.

20. Jongejan, J. A., Duine, J. A., Eds.; PQQ and Quinoproteins; Kluwer: Dordrecht, 1988.

21. Park, W.; Ahmed, J.; Kim, S. Colloids Surf. B: Biointerfaces 2009, $68,120$.

22. Gomez, M. E.; Li, J.; Kaifer, A. E. Langmuir 1991, 7, 1797.

23 Zayats, M.; Katz, E.; Baron, R.; Willner, I. J. Am. Chem. Soc. 2005, 127,12400

24. Matsuda, H.; Aoki, K.; Tokuda, K. J. Electroanal. Chem. 1987, $217,15$.

25. Bulovas, A.; Dirvianskyte, N.; Talaikyte, Z.; Niaura, G.; Valentukonyte, S.; Butkus, E.; Razumas, V. J. Electroanal. Chem. 2006, $591,175$.

26. Laviron, E. J. Electroanal. Chem. 1979, 101, 19.

27. Laviron, E. J. Electroanal. Chem. 1981, 124, 1.

28. Laviron, E. J. Electroanal. Chem. 1981, 124, 9.

29. Laviron, E. J. Electroanal. Chem. 1983, 146, 1.

30. Laviron, E. J. Electroanal. Chem. 1983, 146, 15.

31. Finklea, H. O. J. Phys. Chem. B 2001, 105, 8685.

32. Trammell, S. A.; Lebedev, N. J. Electroanal. Chem. 2009, 632, 127. 